13 | 1991

Varia

\title{
Les ancêtres ambertois de Pascal
}

Michel Boy

\section{OpenEdition}

\section{Journals}

Édition électronique

URL : http://journals.openedition.org/ccibp/626

DOI : $10.4000 /$ ccibp. 626

ISSN : 2493-7460

\section{Éditeur}

Centre international Blaise Pascal

\section{Édition imprimée}

Date de publication : 7 janvier 1991

Pagination : 8-16

ISSN : 0249-6674

\section{Référence électronique}

Michel Boy, «Les ancêtres ambertois de Pascal », Courrier du Centre international Blaise Pascal [En ligne], 13|1991, mis en ligne le 08 janvier 2016, consulté le 19 avril 2019. URL : http:// journals.openedition.org/ccibp/626 ; DOI : 10.4000/ccibp.626

Ce document a été généré automatiquement le 19 avril 2019.

Centre international Blaise Pascal 


\title{
Les ancêtres ambertois de Pascal
}

\author{
Michel Boy
}

1 Marguerite Pascal de Mons, épouse de Martin Pascal, trésorier de France au Bureau des Finances de Riom, l'une des grand'mère de Blaise Pascal, est connue pour être originaire d'Ambert. Mon intérêt, sans doute quelque peu chauvin pour cette région ${ }^{1}, m^{\prime}$ a donc, très naturellement et depuis longtemps, fait trouver irritante l'absorption vellave de cette partie de l'ascendance pascalienne que les recherches de A. de Remacle ne me paraissent pas justifier de façon satisfaisante.

2 Étant aujourd'hui en mesure d'apporter quelques pièces sérieuses et nouvelles à ce dossier, certes mineur, il m'a paru souhaitable de les livrer en priorité aux «Amis du C.I.B.P. ».

3 A. de Remacle a en effet accrédité l'idée d'une origine vellave de Marguerite Pascal de Mons $^{2}$. Certes, on trouve, en Velay, de nombreux Pascal : ceux que cite cet auteur ou dont l'Armorial du Velay dresse la liste ${ }^{3}$, auxquels on pourrait encore ajouter ceux dont les archives de l'abbaye de la Chaise-Dieu gardent la mémoire, comme par exemple : Claude Pascal, juge à la Cour commune du Puy en $1582^{4}$, ou le notaire Jacques Pascal connu en $1516^{5}$, ou bien encore ce Thomas Pascal, curé de Saint-Léger de Sembabel en $1508^{6}$... Certes, on trouve également, au sein de la famille Pascal de Mons, comme le rappelle Ulysse Rouchon ${ }^{7}$, deux chanoines de l'église cathédrale du Puy dans la première moitié du XVI siècle... Mais cela ne suffit pas pour jongler avec les filiations et combler les lacunes de l'information disponible en prenant le relais du facétieux Georges Paul, le regretté auteur de

4 l'Armorial du Velay, si largement sollicité par A. de Remacle.

5 Venons-en donc aux sources documentaires. 


\section{Les frères Pascal, propriétaires ambertois en 1519 : Thomas le Président, Guillaume le Chanoine et Jehan Seigneur de Mons}

6 Un premier document ambertois signale à notre attention la famille Pascal de Mons. Il s'agit du

Terrier et reconnaissance des cens, rentes et aultres droits appartenans à puissant seigneur Jehan de fontanet, escuier, seigneur daulhat, en la chastellenie et mandement dambert (...) icelles reconnaissances reçues par Jehan Laguayte et Jehan solviche, notaires royaux jurés à ambert, soubz le scel de la chancellerie royale de montferrand, entre le 9 mai 1519 et le 19 mai $1523^{8}$.

À la date du 16 mai 1519, comparaît en personne

noble homme Jeahn pasqual, seigneur de mons, tant pour luy que prenant en main pour vénérable et discrète personne monseigneur messire thomas pasqual, docteur ès droitz, conseiller du Roy notre sire et président en sa cour de parlement, et monseigneur guillaume pasqual, licencié en loix, chanoine de notre dame du puy, et aussi pour Jehan petit et damyen pasquaulx ses cousins, enffans à feu gabriel pasqual, pour leur faire avoir agréable et ratiffier, lequel a confessé tenir et ses prédeccesseurs avoir tenu d'ancienneté, du cens, censive et directe seigneurie de noble et puissant seigneur Jehan de fontanet seigneur daulhat à ce présent, c'est à scavoir un certain pré situé au terroir de la tioullarie ès appartances dambert contenant une émine et demye de pré ou entour (...), item certain autre pré contenant deux émines de pré ou entour situé aux appartenances de la ville dambert soubz le mas de vialette (...), item plus une terre contenant troys émynées de terre ou entour, située au terroir de la saigne de baille alias de Layre $^{9} .$.

9 Les terroirs cités sont par ailleurs connus. Las Tiollarias est à l'ouest de la cité près la «charreire publique tendant de la ville au pont» sur la Dore. Le second pré est lui au midi, au-delà du ruisseau de la Maire, près du Petit-Chier. La terre, quant à elle, se trouve au nord-ouest de la ville entre la route d'olliergues et la Dore.

10 On notera que les Pascal tiennent ces biens de leurs prédécesseurs immédiats et vraisemblablement dans l'indivision provisoire, puisque la mention « communs en biens » n'est pas utilisée dans le texte.

11 Ce texte présente donc quelques-uns des membres de deux familles cousines entre elles. D'une part les enfants de feu Gabriel Pascal, dont Jehan dit « le petit », selon une habitude dont le terrier offre maints exemples, pour le distinguer de son homonyme, le seigneur de Mons. D'autre part les trois frères : Thomas, Guillaume et Jehan. 
Les Pascal à Ambert en 1519 : Reconnaissance de Jehan Pascal de Mons, prenant en main pour ses frères Thomas, Président de la Chambre des Enquêtes au Parlement de Paris, et Guillaume, chanoine de Notre-Dame du Puy, et ses cousins Jehan et Damien

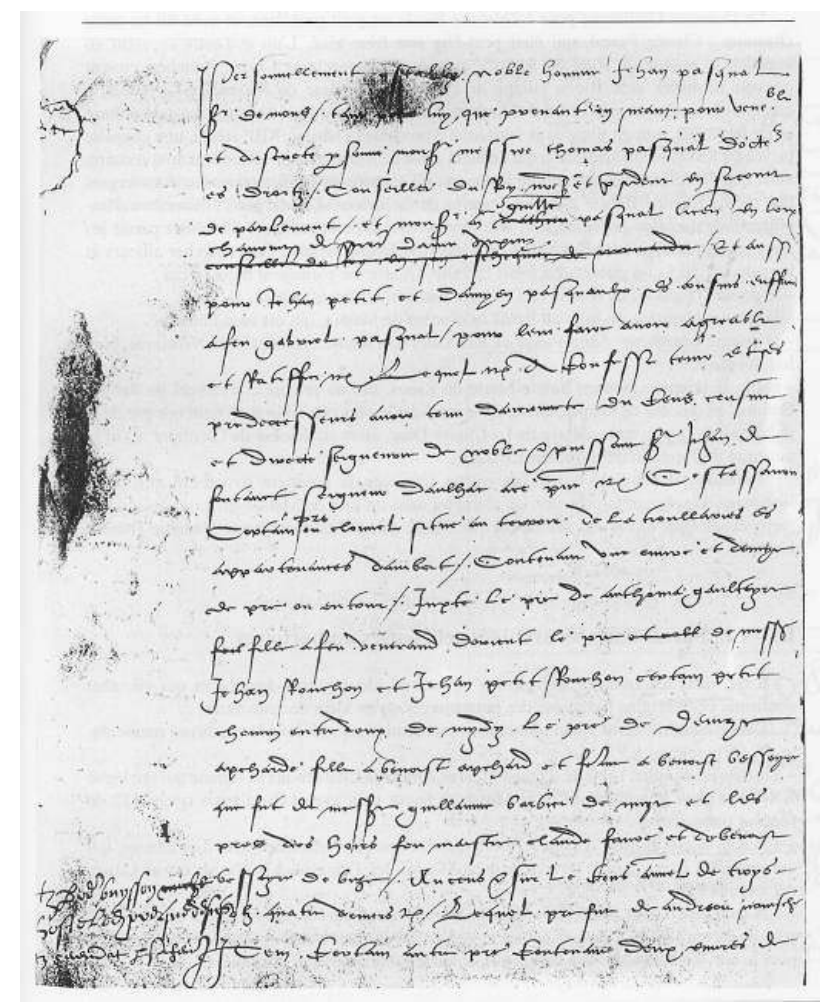

Terrier Fontanet, folio 19 v$^{\circ}$, Archives du Château du Bourgnon. famille, connu lui aussi à Ambert. En effet, au moment de citer le chanoine Guillaume, le notaire-rédacteur avait d'abord écrit : «monseigneur mathieu pascal, licencié en loix, conseiller du Roy en son eschiquier de normandie ». Cette mention, rayée et surchargée, invite à voir dans ce personnage, un autre frère, ou cousin, ou oncle, de ceux que nous venons de rencontrer... D'où on voit qu'Étienne Pascal et ses enfants ne furent pas les premiers de la famille à fréquenter la Normandie ${ }^{10}$ !

17 Le chanoine Guillaume pose à l'histoire locale un petit problème, et avec lui un autre chanoine : Claude Pascal, qui était peut-être son frère aîné. L'un et l'autre reçurent en bénéfice le prieuré de Ruel ou Rieux ${ }^{11}$ que A. de Remacle et Ulysse Rouchon croient pouvoir identifier avec Riols, village de l'actuelle commune de Marsac-en-Livradois, à 
quelques kilomètres au sud d'Ambert. La difficulté est qu'il n'y eut jamais de prieuré en ce lieu mais, tout au plus, dans l'enceinte d'un château bâti au XIII ${ }^{\mathrm{e}}$ siècle, une chapelle privée où les seigneurs de Baffie, qui l'étaient aussi de Riols, avaient fondé leur anniversaire, ainsi que l'enseigne par exemple le testament d'Éléonore de Baffie, comtesse d'Auvergne, que publia Étienne Baluze dans les Preuves de la Maison d'Auvergne ${ }^{12}$. Sans être d'importance la question posée appelle une réponse qu'on peut, semble-t-il, choisir parmi les deux suivantes. Soit faire du prieuré de Riols une simple vicairie. Soit chercher ailleurs et hors du Livradois un prieuré qui ferait l'affaire. Quatre me paraissent dans ce cas :

18 - le prieuré clunisien de Ruelle en Charente malheureusement disparu en 1450.

19 - le prieuré clunisien de Ruel ou Reuil au diocèse de Meaux, qui est bien lointain,

20 - le prieuré bénédictin Saint-Pierre de Riols, uni à la mense épiscopale de Narbonne, bien loin lui aussi,

21 - enfin, le prieuré casadéen Sainte-Marie de Rioux, uni au prieuré conventuel de Sainte Gemme, au diocèse de Saintes. Ce serait le candidat le plus plausible si on tient compte de la proximité de la puissante abbaye de La Chaise-Dieu, alors au diocèse de Clermont et sur le territoire de l'archiprêtré d'Ambert/Livradois.

22 Toutefois, il convient de ne pas rejeter trop vite la première hypothèse qui aurait l'avantage d'expliquer la présence, en réemploi dans un mur de Marsac-en-Livradois, d'une pierre écussonnée aux armes des Pascal... de Mons, qu'a par ailleurs décrite Madame Thérèse Goyet ${ }^{13}$.

\section{Les Pascal dans les archives religieuses ambertoises}

23 II est, dans les archives «religieuses » d'Ambert, deux autres documents qui viennent confirmer cette origine livradoise des personnages qu'on vient de rencontrer.

24 C'est d'abord la moitié d'un obituaire aujourd'hui conservé dans les archives municipales ${ }^{14}$ On y relève :

25 - à la date du 4 juillet, l'obit de «Jean Pascal et Anthonia Chassenona sa femme qui ont légué XX livres pour le service de deux Messes à haute voix avec Libera me » (page 112 de l'ancien obituaire);

26 - à la date du 10 juillet, l'obit de « Noble Gabriel Pascal et Mariette Achard sa femme, qui ont légué XX livres pour le service de deux Messes des Trépassés à haulte voix avec Libera me » (page 115);

27 - à la date du 16 août, l'obit de «Messire Pierre Pascal, curé d'Ambert, qui a légué XXXI sols, un carton soigle, $\mathrm{X}$ cartons avoine et une gelline de cens et rente payable à Sainct Julhen pour le service d'une Messe à basse voix et aussi Libera me et de Profundis ».

28 Le curé Pierre Pascal est connu : il tenait la cure d'Ambert au moment où commençait la construction de la nouvelle église Saint-Jean, en 1471. On aura noté au passage qu'il possédait des terres en censive. Dans la première moitié du siècle suivant, deux autres membres au moins de la famille Pascal devaient lui succéder à la cure : Guillaume connu en 1531 et Damien cité en $1545^{15}$ 
'est pas impossible par ailleurs que Jean Pascal, époux d'Antonia Chassenon, puisse être identifié avec Jean « le petit » du Terrier Fontanet. Il est, en 1536, signalé dans un document du consulat ambertois réglant les mesures de mise en défense de la ville, où il est taxé fournir une hallebarde ${ }^{16}$.

On peut ensuite trouver matière à réflexion dans le texte imprimé d'un mémoire concernant un procès opposant, au début du XVIII ${ }^{\mathrm{e}}$ siècle, le curé d'Ambert et les prêtres communalistes, document fort heureusement conservé dans les archives paroissiales ${ }^{17}$. La communauté des prêtres sociétaires apporte en effet, à l'appui de sa défense, plusieurs écrits vénérables, aujourd'hui introuvables mais cités par le mémoire. Parmi eux, on retiendra:

-« un terrier contenant neuf vingt feuillets (...) finissant par une reconnaissance au profit de ladite communauté consentie par Jehan Pascal, marchand d'Ambert, en datte du 16 juillet $1511 »$ (page 9);

\section{Obit de Jehan Pascal (le 4 juillet)}

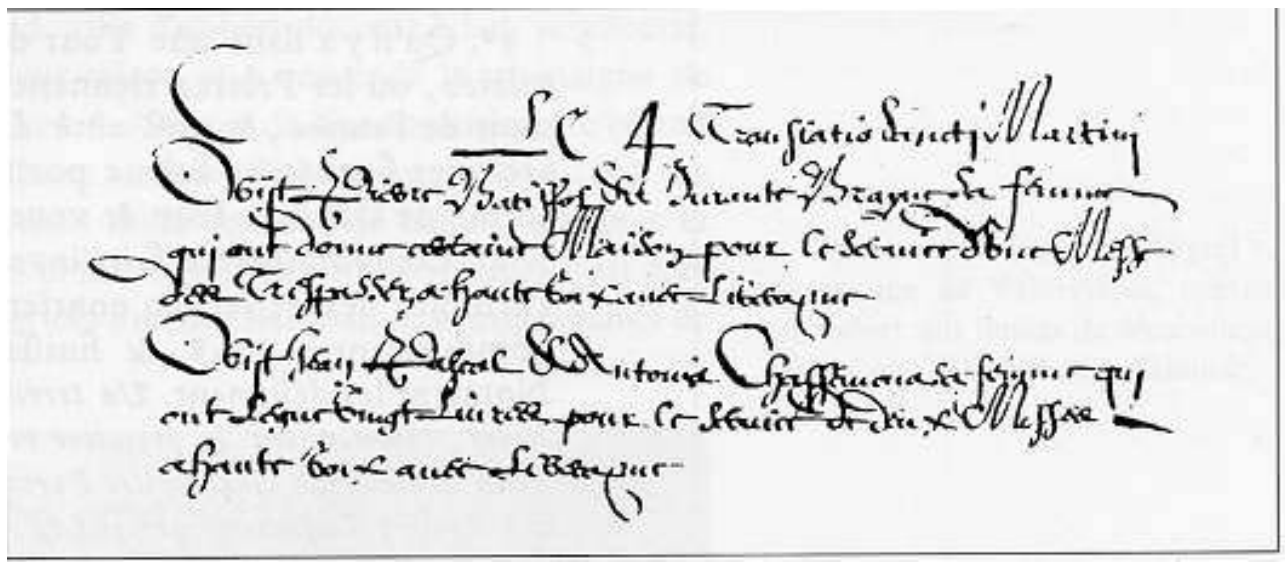

Obit de Gabriel Pascal (le 10 juillet)

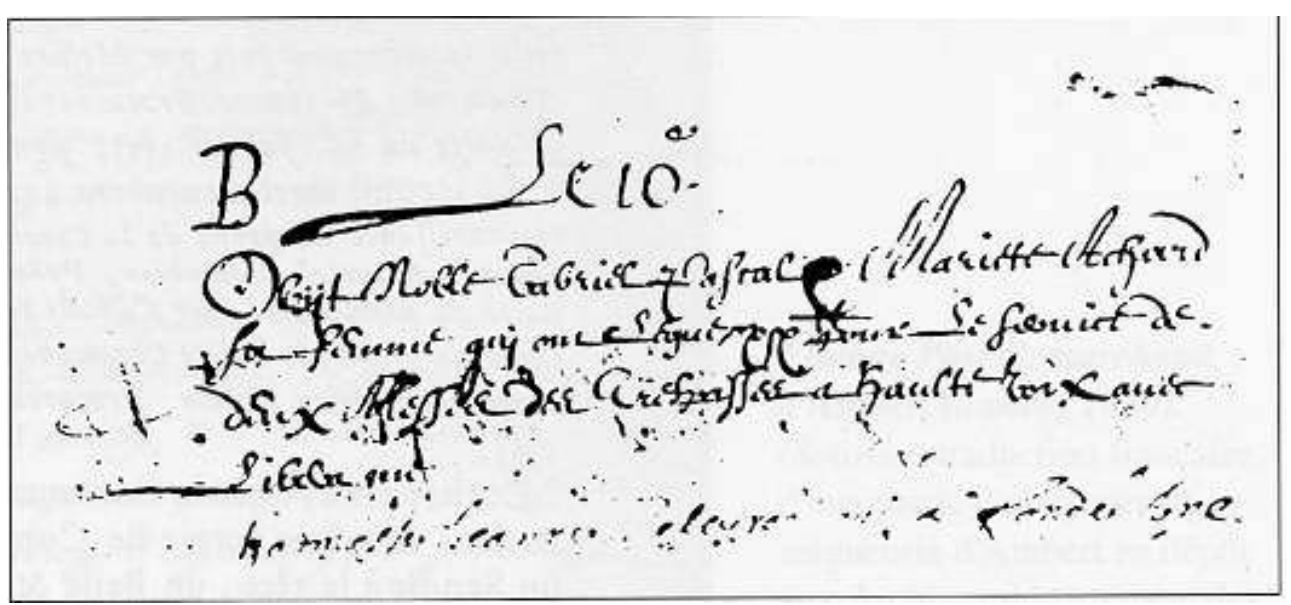

Extraits du « livre des fondations » ou obituaire de l'église Saint-Jean d'Ambert

Source : Archives municipales d'Ambert, État civil, registre troisième 
Obit de Pierre Pascal, curé d'Ambert (le 16 août)

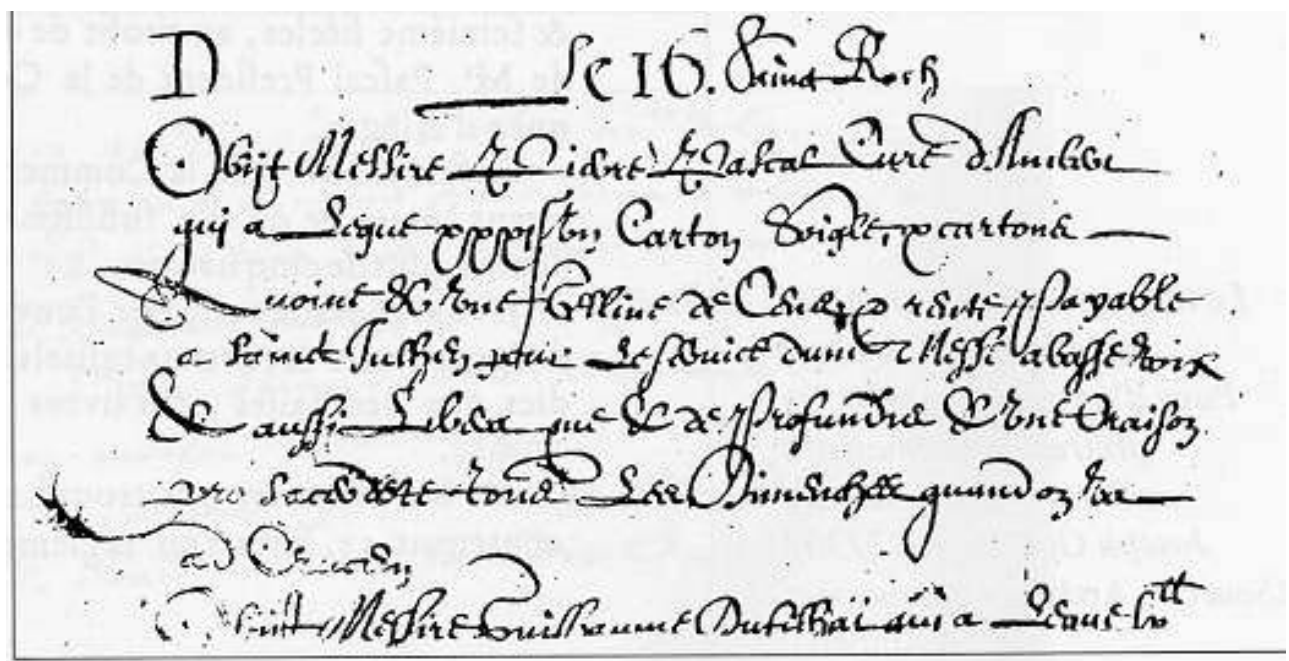

Extraits du « livre des fondations » ou obituaire de l'église Saint-Jean d'Ambert

Source : Archives municipales d'Ambert, État civil, registre troisième

-« la fondation de Vespres faite en 1527 par M. Pascal, Président des Enquêtes à la Cour » (page 10).

Tous ces documents qui avaient échappé aux guerres religieuses, étaient alors conservés dans les salles basses du clocher de l'église Saint-Jean-Baptiste. Ils sont sans doute, aujourd'hui, irrémédiablement perdus.

Il en va de même des documents consultés par l'ambertois Benoît-Marie Madur-Dulac, dans un projet resté manuscrit d'Histoire d'Ambert ${ }^{18}$. Ce dernier fait état de diverses fondations de messes par le Président Thomas Pascal, qui les avait assises sur les revenus de plusieurs maisons du quartier du Pontel, dont l'une confinait la maison de Gabriel Bellot dont un fils devait présider le Parlement de Rouen ${ }^{19}$ Les documents consultés se trouvaient alors dans les archives municipales d'où ils ont disparu avec beaucoup d'autres... 


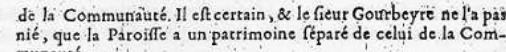
$2^{\circ}$. Que la Communauté a dans la chapelle de Saint Pierré ancieinnes archives fermantes à trois portes, re de titres.

nour du Clocher une Chambre Capis culaire, où les Prêtres tiennent leur A fiemblée generale tous les mois de l'année, \& qu'à côrté de cette Chambre, il y a d'aurr: archives fermantes a deux portes faites depuis plus d'un fiecle $c$ forme de Cabinet fort $\alpha$ voute.

1. $4^{\circ}$. Les Notaites ont firt inventaire des titres, \& il paroît que commentente Notaires les défignent. Un terrier contenant neuf vingt fix fenillets écriss, commengant la premiere reconnoiffante en latin, confentic par Fean Rolbon de Gratarelles Paroije d clmberg, au profit des Pyetres des Eglifes d Amberg prefent o acceptant Andre Cboriat Trant

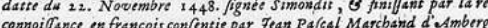
Lidis enserinement fais par zAefire Pierre Bertonnee de Valleyra PrE tre at som es comme Procureur de la Communause de ladiste Eglife, en datse du 16. Fuillet istis. fgené Debonnaire. Le fecond terrier contient 310 . feüillets, commençant la premier

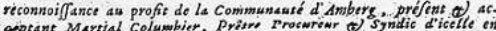

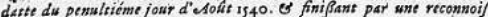
Sance as profit de ladite Comminnaute présents Maftres fean Vernet Antoine Andrieu Prétre, Procureur, Baile of Syndic dit 3. Ottobre Is65. $\&$ dans la même forme de Communaute qu'aujourd hui, avec un Syndic à la tête,

dans le quinziéme A de M'. Pafcal Prefident de la Cour, \& de noble. Seigneur Jac-

ques d'Alegre. ferens tems, \& qu'elle fubrifte comme Corps de Communaute depuis plus de cing fíćcles.

$3^{\circ}$ Les Notaires ont fait l'ouverture des Livres de diftribution des gros fruits \& revenus cafuels de la Communauté, tantdes Syn

quinze. contenant 33. fatutsout reglemens en latin dont le premier con.

Page d'un mémoire pour les prêtres communalistes ambertois contre le curé Joseph Gourbeyre (1730).

Source : Archives paroissiales d'Ambert.

\section{L'assiette de rente de 1497-1502}

Il ne semble pas possible de confondre le Jehan Pascal, marchand d'Ambert, cité ci-dessus en 1511, et alors sans doute proche de sa fin, avec Jehan, seigneur de Mons, ou encore Jehan dit « le petit ». Il s'agit bien plutôt du père du sieur de Mons, du Président Thomas et du chanoine Guillaume. C'est du moins ce qu'on est en droit de conclure à la lecture de la « déclaration par le menu des cens, rentes et autres droitz et devoirs de la seigneurie dambert par damoyselle Anthonia de Polignac, vesve de feu Godeffroy de La Tour, en son vivant seigneur de Montgascon et du pays de Livradoys ", aujourd'hui conservée aux Archives nationales ${ }^{20}$.

$\mathrm{Au}$ folio 357 de ce volumineux terrier, dans la liste de «ceulx qui tiennent dans lad. chastellenie dambert fiefs et hommaiges », apparaissent « Jehan et Gabriel Pasqualx (qui) tiennent en fief la somme de trente livres de rente ».

On retrouve ces deux personnages, dont on apprendra qu'ils sont frères, cités ensemble ou séparément dans bon nombre de reconnaissances. Ainsi, au nord d'Ambert, près du village de Combris où «Jehan et Gabriel Pasquals, frères, enfans et héretiers de feu Estienne Pasqual, marchand dambert », disposent de beins importants. Ils sont encore très présents dans la paroisse voisine de Valcivières où ils sont signalés sur les terres des villages de La Girynie (aujourd'hui La Jurinie) et de Montauroux. Surtout « Maistre Jehan Gailhard, notaire de la ville dambert et Gabriel Paschal, bourgeois de lad. ville dambert 
doivent à lad. seigneurie, chacun an, la somme de cinquante livres (...) pour raison et à cause de la montaigne de Puygros avec ses appartenances quelzcunques (...) en tout droict de directe seigneurie et tout selon la forme et teneur de la vestizon à eulx faicte » (folio 214 verso et folio 215). La Montagne de Puygros (aujourd'hui Pégrol) c'est trois mille setérées de landes, bruyères et herbages d'altitude, propres à paître, en été, vaches et moutons. Trois mille setérées c'est près de deux mille de nos hectares, que nos riches ambertois louent à leur tour aux propriétaires de bétail pour l'estive ${ }^{21}$.

Étienne Pascal, marchand d'Ambert (années 1460).

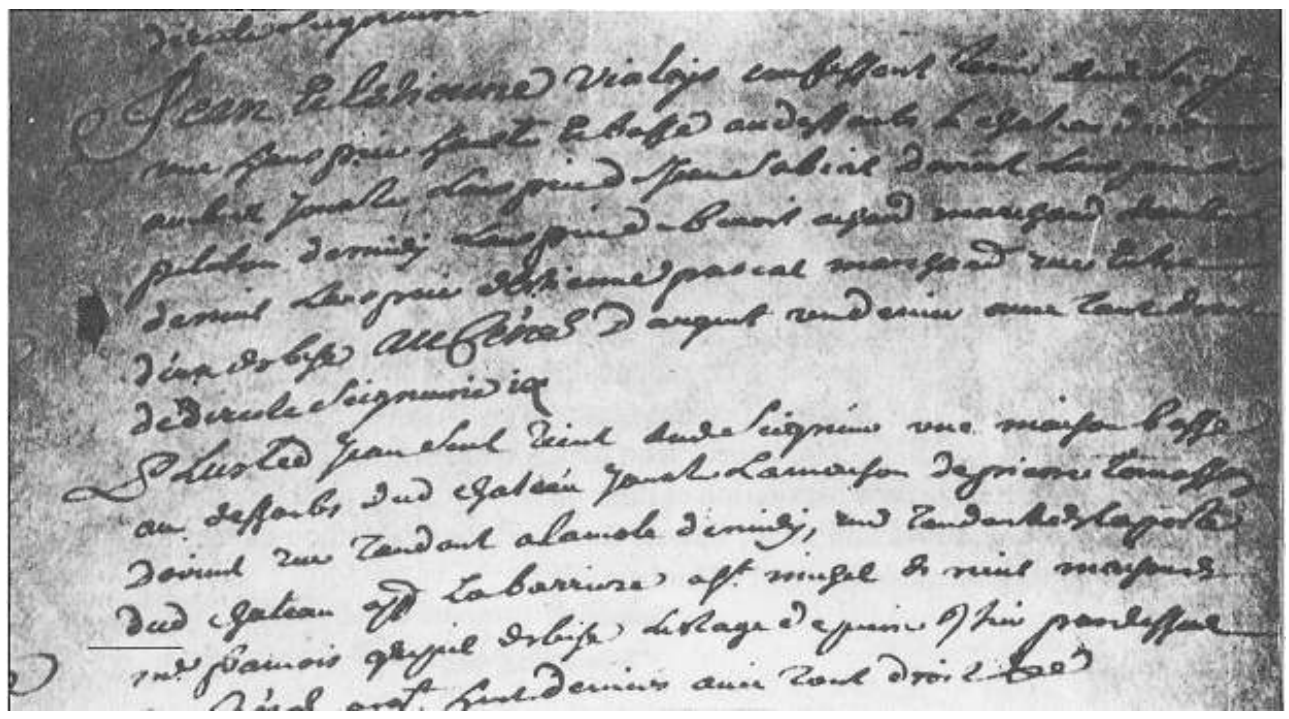

Source : traduction française d'une partie d'un terrier de la seigneurie d'Ambert en dépôt aux Archives départementales du Puy-de-Dôme.

Traduction française d'une partie d'un terrier de la seigneurie d'Ambert en dépôt aux Archives départementales du Puy-de-Dôme

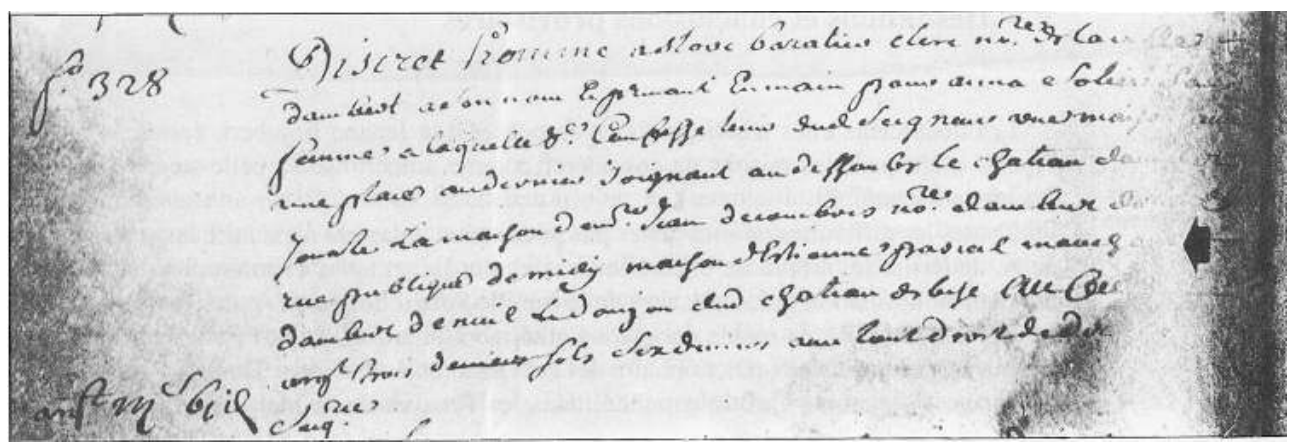

Archives départementales du Puy-de-Dôme

Ainsi, à travers les documents évoqués, apparaît plus clairement l'ascension sociale de la famille Pascal, à la charnière des $\mathrm{XV}^{\mathrm{e}}$ et $\mathrm{XVI}^{\mathrm{e}}$ siècles. Gabriel, simple marchand, titré de «bourgeois » en 1500, se verra donner du «noble » dans l'obituaire paroissial. Noble, il ne l'est peut-être pas vraiment. Mais il tient déjà en fief, avec son frère Jean, pour trente livres de rente dans le châtellenie d'Ambert et il loue à la dame du lieu, la Montagne de Pygros pour cinquante livres l'an. Ce qui n'est pas rien. Autre signe : Gabriel a pris épouse dans la famille Achard, famille de riches notables, titulaires du fief de Chicot ${ }^{22}$. 

branche aînée, d'où devait précisément sortir Marguerite Pascal de Mons, grand'mère paternelle de Blaise Pascal.

\section{IV. Étienne Pascal, marchand à Ambert dans les années 1460}

Étienne Pascal, père de Jehan et Gabriel Pasquaulx, ne nous est pas inconnu. Il apparaît dans plusieurs reconnaissances d'une copie, malheureusement partielle, d'un autre terrier de la seigneurie d'Ambert aujourd'hui déposé, semble-t-il, aux Archives départementales du Puy-de-Dôme ${ }^{23}$.

$41 \mathrm{Au}$ folio $328 \mathrm{du}$ terrier ancien, "discret homme astorc baratier, notaire de la ville dambert (...) confesse tenir dud. seigneur une maison avec place derrier au dessoubs le château dambert, jouxte la maison de $\mathrm{M}^{\mathrm{e}}$ Jean decombres, notaire dambert dorient, rue publique de midy, maison dEstienne pascal, marchand dambert de nuyt, le donjon dud. château de bise ». Cette même maison d'Etienne Pascal fait vis-à-vis, de l'autre côté de la rue, à celle de Jean et Estienne Vialeys, dont la reconnaissance est consignée au folio 347.

D'autres articles mentionnent encore Étienne Pascal mais aucun ne le concerne directement. Aussi est-il impossible de préciser si la maison mention née ci-dessus, est sa résidence principale. Si tel était le cas, les indications topographiques fournies par l'ensemble du terrier permettraient de la situer dans la partie haute de l'actuelle Rue du Château et vraisemblablement à la hauteur du numéro 23.

43 Ce texte livre également le nom, au folio 252 verso, de "vénérable et discret homme maître pierre pascal, prêtre, bachelier en droit canon, recteur et curé de léglise dambert ", déjà signalé et qui paraît bien appartenir à la même génération que le marchand Étienne dont il pourrait être le frère ou le cousin.

Il me semble par ailleurs possible de reconnaître dans Jehan Pascal, fils du marchand Etienne et père du Président Thomas, l'un des quatre consuls ambertois en charge l'an 1484. En effet, lorsque, le 3 août de cette année, Jean de La Tour, seigneur d'Ambert, se présente devant la ville pour en prendre possession et jurer de respecter ses franchises, il est accueilli par « honorables hommes Jean Pascal, Benoît Gauthier, Pierre Mathon et Jean Delagoutte, consuls l'année présente de ladite ville d'Ambert ${ }^{24}{ }^{2}$.

\section{Hésitations et conclusions provisoires}

Les documents cités n'invitent-ils pas à penser que Jeanne Enjobert, épouse d'Étienne Pascal, avait quelques raisons de considérer comme ambertoise sa belle-sœur Marguerite Pascal de Mons ${ }^{25}$ ?

Toutes les difficultés ne sont certes pas pour autant aplanies. À prendre les seules études de A. de Remacle, il faudrait encore lever quelques incertitudes et contradictions touchant notamment la filiation et les alliances de la famille Pascal de Mons. A. de Remacle attribue en effet, semble-t-il, la même épouse : Catherine Guitard de Saint-Privat, tantôt à Jehan Pascal, seigneur de Mons (Dictionnaire des fiefs), tantôt au Président Thomas Pascal (article cité, page 8). Antoine Jacotin a publié, dans les Preuves de la Maison de Polignac, une obligation consentie par Robert de Rochebaron pour le vicomte

Courrier du Centre international Blaise Pascal, 13 | 1991 
de Polignac, au profit de « noble François Pascal de Mons ». La dite obligation s'élève à 70 écus, à raison d'une cédille de 60 écus concernant son oncle: «feu maître Guillaume Pascal prieur de Rieux », et du remboursement d'une dépense de dix autres écus engagée par « ledict Pascal et damoyselle Catherine de Saint-Privat sa mère ${ }^{26}$ ». Ce texte paraît clair. Mais alors, celui qui retient la leçon, peut-être hâtive, de A. de Remacle, doit faire de François Pascal le fils du Président Thomas. Ce qui remet en question l'article du Dictionnaire des fiefs qui le fait fils de Jeahan Pascal, seigneur de Mons... Sauf, bien sûr, à supposer deux Catherine parfaitement homonymes.

Terrier de la seigneurie d'Ambert, prenant sur Saint-Martin, Champetières, Notre-Dame de Mons, Saint-Ferréol et Valsivières

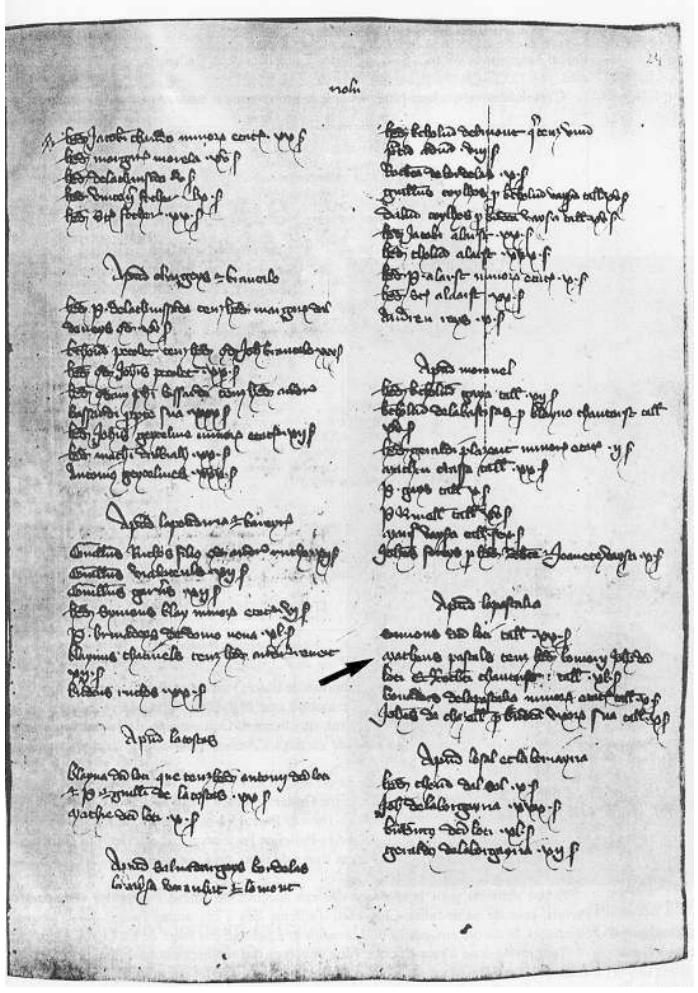

Rédigé entre 1462 et 1471. Anciennes archives privées de M. France Durif. Microfilm aux Archives Départementales sous la cote I.MI.166.

C'est donc avec quelque prudence que je proposerais le tableau généalogique suivant : 
Tableau généalogique d'Étienne Pascal

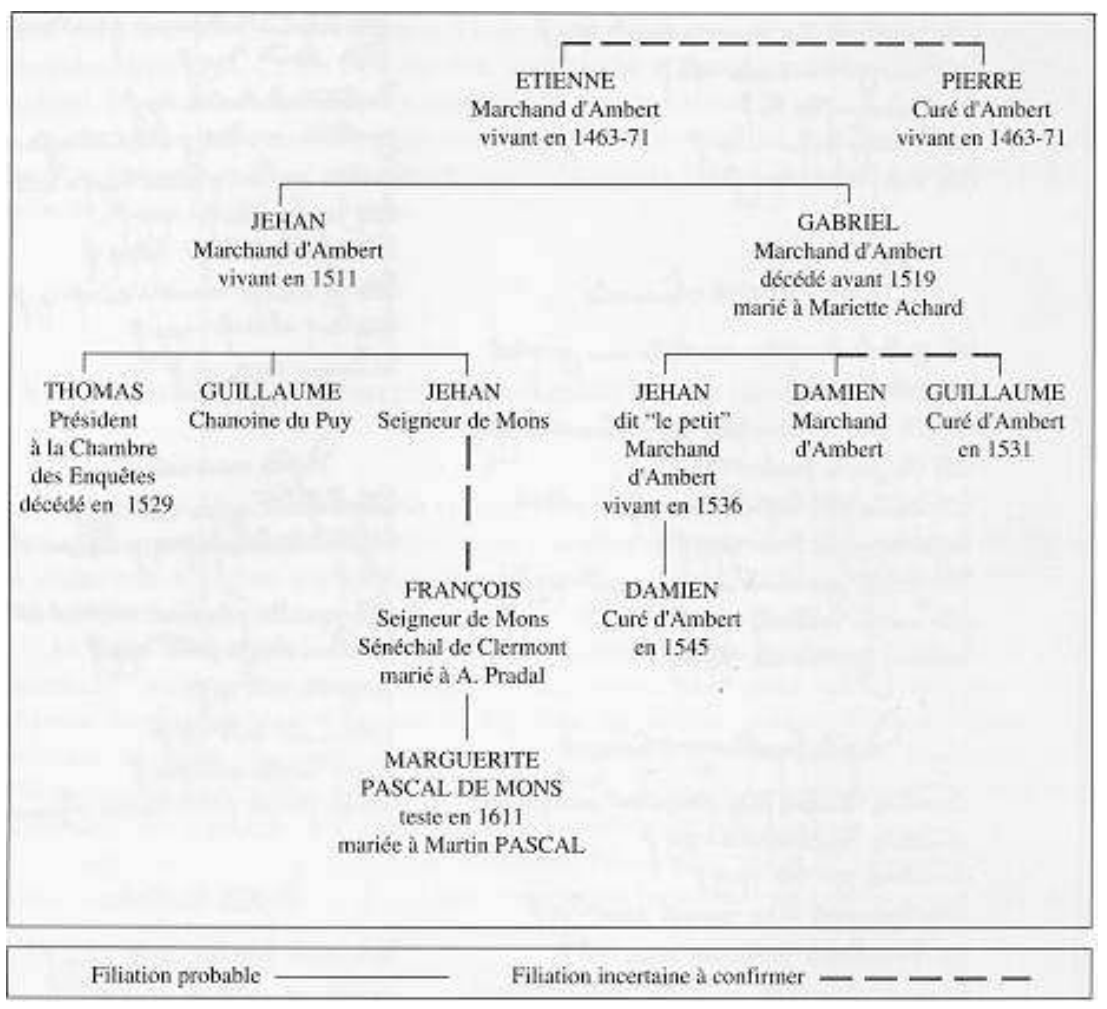

En portant à la connaissance des familiers de Blaise Pascal ces quelques textes empruntés à l'Histoire ambertoise, j'espère avoir montré que l'ascendance de Marguerite Pascal de Mons, celle du Président Thomas Pascal, du chanoine Guillaume et de Jehan, seigneur de Mons, paraît bien s'enraciner fortement en pays d'Ambert, tout autant et plus qu'en terre vellave.

Que la famille Pascal de Mons ait eu des attaches avec le Velay, c'est indéniable si, comme le pense A. de Remacle, Catherine Guitard est bien Dame de Saint-Privat-d'Allier, à quelques kilomètres à l'ouest du Puy ${ }^{27}$. Thomas Pascal lui-même avait sans doute profité de ces liens avec le Velay et les Chalencon-Polignac pour acquérir, du vicomte Guillaume Armand, décédé en 1518, quatre-vingts livres de rente sur la seigneurie de Beaumont, aux portes du Livradois ${ }^{28}$.

Est-ce suffisant pour faire descendre ces ancêtres de Blaise Pascal des seigneurs du Pertuis, près de Saint-Julien-Chapteuil, d'ailleurs liés à ces autres Pascal qu'on trouve à Sembabel, là encore aux portes du Livradois et à l'ombre des tours de La Chaise-Dieu ${ }^{29}$ ?

51 Tout invite donc à rappeler que l'une des tours qui renforçaient les murailles d'Ambert, ainsi que l'une des poternes qu'elle surveillait, portaient le nom des Pascal ${ }^{30}$. En 1536 pourtant, la « Tour Pascal » n'était plus « jouie et possédée » par un membre de la famille, mais par «les héritiers de Jacques Boysson ${ }^{31}$. C'est que, sans doute, portés par leur ascension sociale, les Pascal étaient en passe de quitter Ambert pour d'autres cieux, même s'ils gardaient intact le souci d'y fonder leur souvenir, à l'image du Président Thomas.

Un mot encore. L'imprudence aidant, je rappellerai que, non loin de Marsac-en-Livradois où l'on a retrouvé la pierre sculptée de l'agneau pascal, il existe, sur le territoire de l'actuelle commune de Beurières, un hameau appelé La Pascaille. Ce nom est l'aboutissement d'un ancien La Pascalia, connu dès 1350 et qui était alors la résidence 
ordinaire d'un certain Mathieu Pascal qu'on pourra tenir, au moins provisoirement, pour le plus ancien Pascal repéré en pays d'Ambert ${ }^{32}$.

\section{NOTES}

1. Boy (Michel), Ambert, deux mille ans d'histoire, Limoges, 1983.

2. Remacle (A. de), «Les Pascal en Basse-Auvergne», dans : Blaise Pascal, quelques souvenirs sur lui et les siens, Clermont-Ferrand, 1923.

3. Paul (Georges), Armorial du Velay, Paris, 1912, pp. 332-333. Cet ouvrage est largement sollicité par A. de Remacle.

4. Archives départementales de la Haute-Loire, 1.H. 201.

5. Ibidem, 1. H. 325.

6. Ibidem, 1. H. 12, folio 112.

7. Rouchon (Ulysse), «Un arrière grand-oncle de l'auteur des Provinciales, Guillaume Pascal, chanoine du Puy au XVI ${ }^{\mathrm{e}}$ siècle», dans : Bulletin historique, scientifique..., publié par la Société académique du Puy, 1929, XVI, p. 92.

8. Archives du château du Bourgnon (Puy-de-Dôme), obligeamment mises à notre disposition par M. Charles Micolon de Guerines. Aulhat est Aulhat-Saint-Privat, canton et arrondissement d'Issoire. Sur ce terrier et ce fief, on peut voir : Charles Micolon de Guerines, « La maison forte, métairie noble et seigneurie de Blanval", dans Chronique historiques d'Ambert et de son arrondissement, numéro hors-série consacré au canton de Saint-Amant-Roche-Savine, 1990, pp. 31 ss.

9. Terrier Fontanet, folio $19 \mathrm{v}^{\circ}$. Pour l'anecdote, on se souviendra que l'éminée ou éminade (qui est la moitié de la setérée) représentait, à Ambert, une trentaine de nos ares. La setérée valait en effet 1600 toises carrées.

10. Mathieu Pascal est cité dans d'autres documents. Il peut simplement paraître curieux qu'il soit ici parlé de l'Échiquier de Normandie car il était devenu, en 1499, le Parlement de Rouen.

On y trouvera, au XVI ${ }^{\mathrm{e}}$ siècle, d'autres Auvergnats.

11. Jacotin (A.), Preuves de la Maison de Polignac..., pièce 221 pour « Claude Pascal, prieur de Ruel » en 1529 , et pièce 229 pour « Guillaume Pascal, prieur de Rieux » en 1546.

12. Baluze (Etienne), Preuves de la Maison d'Auvergne, p. 117 ss.

13. Cette pierre armoriée a été publiée par Thérèse Goyet dans le Courrier du CIBP., 8, 1986, p. 25.

14. Archives municipales d'Ambert, état-civil, registre $n^{\circ}$ 3. Sur ce document, voir Michel Aman, «Le nécrologe de l'Église Saint-Jean d'Ambert», dans le Bulletin historique et scientifique de l'Auvergne, 1985, p. 359 ss.

15. Boy (Michel), Ambert, deux mille ans d'histoire déjà cité, annexes.

16. Archives municipales d'Ambert. Sur ce document voir Michel Boy, «La mise en défense d'Ambert en 1536 », dans : Chroniques d'Ambert et de son arrondissement, $\mathrm{n}^{\circ}$ 14, 1992.

17. «Mémoires pour les Prêtres, Syndic et Communauté de l'Église de Saint Jean d'Amberg (sic), Intimez et Défendeurs, contre $\mathrm{M}^{\mathrm{e}}$ Joseph Gourbeyre, Prêtre et Curé de l'Église Saint-Jean de la dite ville d'Amberg, Appelant et Demandeur.» Sur ce document, voir Michel Boy, «La Communauté des prêtres filleuls d'Ambert ", dans : Chroniques d'Ambert et de son arrondissement, $\mathrm{n}$ - 7, 1985, pp. 75-75. 
18. Archives privées de feu Maître Jean Banière, d'Ambert. Benoît-Marie Madur-Dulac fut maire d'Ambert en 1815-1816.

19. Étienne Bellot, au soir de sa vie, aumôna le nouvel hôpital de sa ville natale ainsi qu'une Confrérie charitable sous le titre de Saint-Etienne. Cet hôpital reçut ses lettres patentes le 5 juillet 1554.

20. Archives nationales, Q.1* 930. Ce recueil volumineux a été rédigé après la mort de Godefroy de La Tour en 1497. Mais il évoque également des reconnaissances antérieures qui s'étalent, dates extrêmes, entre 1482 et 1502 . Je remercie ici $\mathrm{M}^{\mathrm{me}}$ A. Vallée, Conservateur des archives modernes aux Archives nationales, qui a bien voulu revoir les références aux membres de la famille Pascal sur l'original.

21. La "Montagne de Pégrol ", commune de Valcivières, canton d'Ambert, aux limites du département. de la Loire, à 1400 mètres d'altitude.

22. Ainsi, Jehan et Benoît Apchard apparaissent-ils dans la même liste de ceux qui tiennent fiefs dans la châtellenie d'Ambert.

Voir également : "Jehan Achard, Claude Faure et Benoît Achard, communs en biens en ceste partie en toutes choses, ont affirmé par serment quilz tiennent XV livres de rante ou un peu moins", Aveux et dénombrements des nobles et roturiers tenant fiefs (...) en 1488 », publiés par E. Teilhard de Chardin dans: Bulletin historique et scientifique de l'Auvergne, 1889.Ce fief avait été constitué à la fin du XV siècle par la famille de Tourzel d'Allègre, et comportait, outre quelques terres, une vicairie attachée à une chapelle de l'église paroissiale.

23. Terrier de la seigneurie d'Ambert, prenant sur Saint-Martin, Champetières, Notre-Dame-deMons, SaintFerréol et Valcivières, rédigé entre 1462 et 1471. Anciennes archives privées de M. France Durif (microfilm aux Archives départementales sous la cote 1.MI.166).

24. Boudet (Marcelin), "Collection inédite de chartes de franchises de Basse-Auvergne ", dans : Mémoires de l'Académie de Clermont, 1914, p. 87.

25. Il s'agit bien sûr de Jeanne Enjobert (1561-1641), épouse d'Étienne Pascal, marchand de Clermont, lequel était frère puiné de Martin Pascal, Trésorier de France à Riom, époux de Marguerite Pascal de Mons.

26. Jacotin (Antoine), Preuves..., t. I, p. 221, pièce 276.

27. Article cité.

28. Jacotin (Antoine), Preuves..., t. 1, p. 221, pièce 238. Il s'agit d'une quittance de 1529, donnée par les exécuteurs testamentaires de feu le Président Pascal, dont son frère Claude, chanoine de Notre-Dame du Puy et prieur de Ruel.

Beaumont est aujourd'hui un simple hameau de la commune de SaintVictor-sur-Arlanc. Il fut autrefois le siège d'une seigneurie et appartenait, sous l'ancien régime, au diocèse de Clermont et à l'archiprêtré de Livradois

29. Jean-Tassin Pascal, seigneur du Pertuis-en-Velay, est témoin le 20 octobre 1566, d'une donation faite par Robert Pascal, hôte de Sembadel, à sa femme, Anne de Fretat et à leurs filles (Archives départementales du Puy-de Dôme, Registres des insinuations de la Sénéchaussée de Riom, $\left.\mathrm{n}^{\circ} 2, \mathrm{f}^{\circ} 418\right)$. On ajoutera, déjà signalé, Thomas Pascal nommé à la cure Saint-Léger de Sembadel en mars 1508... Ou encore : "Jehan Pascal de Sainct Babel en Aulvergne », protestant réfugié à Genève en 1556 (Michel Boy, «Le premier Protestantisme auvergnat et le Refuge de Genève ", dans : Bulletin historique et scientifique de l'Auvergne, 1981, p. 333 sq.).

30. Archives communales d'Ambert: Mesures de défense arrêtées par le Consulat ambertois en 1536 (Michel Boy, Ambert, deux mille ans d'Histoire, p. 76-78 et 102-105).

31. On notera qu'en marge du folio 19 verso du Terrier Fontanet, un scribe a signalé le transfert de propriété du pré de Las Tioleyras des frères Pascal à un certain Pierre Buysson (qui est le même nom que Pierre Boysson).

32. Archives nationales, R.2* 3 1, Registre de levée de taille au profit de la reine Jeanne de France, comtesse d'Auvergne et Dame d'Ambert et du Livradois, f $\mathrm{f}^{\circ} 24 \mathrm{r}$. 
INDEX

Mots-clés : Pascal, généalogie

Index géographique : Ambert 